\title{
A SEMI-FREDHOLM PRINCIPLE FOR PERIODICALLY FORCED SYSTEMS WITH HOMOGENEOUS NONLINEARITIES
}

\author{
A. C. LAZER AND P. J. MCKENNA \\ (Communicated by Kenneth R. Meyer)
}

\begin{abstract}
We show that if the potential in a second-order Newtonian system of differential equations is positively homogeneous of degree two and positive semidefinite, and if the unforced system has no nontrivial $T$-periodic solutions $(T>0)$, then for any continuous $T$-periodic forcing, there is at least one $T$-periodic solution.
\end{abstract}

In this note we consider a system of second-order differential equations in which the nonlinear terms have one nice property shared by linear functions: they are positively homogeneous of degree one. One such system arises from an idealized model of a suspension bridge considered previously in [4 and 7].

Consider the partial differential equation

$$
U_{t t}+a^{2} U_{x x x x}+k(x) U^{+}=f(x, t),
$$

where $U=U(x, t), 0 \leq x \leq L$, with boundary conditions

$$
U(0, t)=U(L, t)=U_{x x}(0, t)=U_{x x}(L, t)=0 .
$$

If $k(x) \equiv 0$, we have the well-known equation for the transverse vibrations of a beam of length $L$, which is hinged at the endpoints, subject to an external force given by $f(x, t)$. If the beam is suspended from above by cables, whose strengths may vary from point to point, and the displacement $U$ is measured in the downward direction, we add the term $k(x) U^{+}$, where $k(x) \geq 0$ and $U^{+}$is the positive part of $U$. This accounts for the fact that cables exert no restoring force when slack.

When $f$ is periodic in $t$ it is natural to look for time-periodic solutions. We consider an approximate problem obtained by discretizing in the space variable $x$. If $N$ is a large positive integer and we consider the mesh points $x_{k}=k L / N$, $k=1, \ldots, N-1$, we are lead to the system of ordinary differential equations

$$
u^{\prime \prime}(t)+A u(t)+D u(t)^{+}=p(t),
$$

Received by the editors March 27, 1983 and, in revised form, May 31, 1988.

1980 Mathematics Subject Classification (1985 Revision). Primary 34C25.

Key words and phrases. Leray-Schauder continuation method, periodic solution.

The first author was partially supported by NSF under Grant DMS9519882.

The second author was partially supported by NSF under Grant DMS8519726. 
where $u(t)$ is the $(N-1)$-dimensional column vector whose $k$ th component is $U\left(x_{k}, t\right), p(t)$ is the vector with $k$ th component $f\left(x_{k}, t\right), u(t)^{+}$has its $k$ th component equal to $U\left(x_{k}, t\right)^{+}, D$ is a diagonal matrix with nonnegative entries, and $A$ is the symmetric, positive-definite matrix corresponding to the finite difference approximation to $U_{x x x x}$, taking into account the boundary conditions. We consider a more general class of systems.

Let $V \in C^{1}\left(\mathbf{R}^{n}, \mathbf{R}\right)$ be positively homogeneous of degree two, i.e., $V(t x)=$ $t^{2} V(x)$ for $t \geq 0$ and $x \in \mathbf{R}^{n}(n \geq 1)$. If, for brevity, we set $V^{\prime}(x)=$ $\operatorname{grad} V(x)$, then $V^{\prime}$ is positively homogeneous of degree one, i.e.,

$$
V^{\prime}(t x)=t V^{\prime}(x)
$$

for $t \geq 0$ and $x \in \mathbf{R}^{n}$. If $($,$) denotes the usual inner product on \mathbf{R}^{n}$, then for Euler's identity we have

$$
\left(x, V^{\prime}(x)\right)=2 V(x)
$$

for $x \in \mathbf{R}^{n}$.

Theorem 1. Let $V \in C^{1}\left(\mathbf{R}^{n}, \mathbf{R}\right)$ be positively homogeneous of degree two and positive semidefinite, i.e.,

$$
x \in \mathbf{R}^{n} \Rightarrow V(x) \geq 0
$$

Let $T>0$. If the system

$$
u^{\prime \prime}(t)+V^{\prime}(u(t))=0
$$

has no $T$-periodic solution other than $u(t) \equiv 0$, then for any $T$-periodic function $p \in C^{1}\left(\mathbf{R}, \mathbf{R}^{n}\right)$ the system

$$
u^{\prime \prime}(t)+V^{\prime}(u(t))=p(t)
$$

has at least one $T$-periodic solution.

Proof. We first assume the stronger condition that $V$ be positive definite, i.e.,

$$
x \in \mathbf{R}^{n}, \quad x \neq 0 \Rightarrow V(x)>0 .
$$

We first show that if $p$ and $V$ are as above, then for $\varepsilon>0$ the system

$$
u^{\prime \prime}(t)+\varepsilon u^{\prime}(t)+V^{\prime}(u(t))=p(t)
$$

has at least $T$-periodic solution. To do this we use the Leray-Schauder continuation method (see, for example, [1 or 6]). Let || denote the usual Euclidean norm on $\mathbf{R}^{n}$, and let $C_{T}$ and $C_{T}^{1}$ denote the Banach spaces of $T$-periodic functions which are continuous and continuously differentiable respectively with norms

$$
\begin{array}{ll}
|v|_{\infty}=\sup _{[0, T]}|v(t)|, & v \in C_{T}, \\
|v|_{1}=|v|_{\infty}+\left|v^{\prime}\right|_{\infty}, & v \in C_{T}^{1} .
\end{array}
$$

Since the second-order linear homogeneous differential system

$$
u^{\prime \prime}(t)+\varepsilon u^{\prime}(t)+u(t)=0
$$


$\left(u \in \mathbf{R}^{n}\right)$ has no $T$-periodic solution other than $u(t) \equiv 0$, it follows that, for every $f \in C_{T}$, there exists a unique $T$-periodic solution of

$$
u^{\prime \prime}(t)+\varepsilon u^{\prime}(t)+u(t)=f(t) .
$$

Moreover, if we denote the unique $T$-periodic solution of the last system by $K(f)$, then $K$ may be viewed as a compact linear operator from $C_{T}$ into itself. Let $N: C_{T} \rightarrow C_{T}$ be the completely continuous operator defined by $N(u)=K\left(u+p-V^{\prime}(u)\right)$. We claim that there exists a number $R>0$ such that if $\lambda \in[0,1]$ and $u \in C_{T}$, then

$$
u=\lambda N(u)
$$

implies that $|u|_{\infty} \leq R$.

Since (1.7) holds if and only if

$$
u^{\prime \prime}(t)+\varepsilon u^{\prime}(t)+(1-\lambda) u(t)+\lambda V^{\prime}(u(t))=\lambda p(t),
$$

and since $|u|_{\infty} \leq|u|_{1}$ if $u \in C_{T}^{1}$, assuming that the above claim is false, we infer the existence of a sequence $\left\{u_{m}\right\}_{1}^{\infty}$ and a corresponding sequence of numbers $\left\{\lambda_{m}\right\}_{1}^{\infty}$ such that $u_{m}(t)$ is a solution of (1.8) when $\lambda=\lambda_{m}$ for $m=1,2, \ldots, \lambda_{m} \in[0,1]$, and

$$
\left|u_{m}\right|_{1} \rightarrow \infty \text { as } m \rightarrow \infty \text {. }
$$

Setting $w_{m}(t)=u_{m}(t) /\left|u_{m}\right|_{1}$ for $m=1,2, \ldots$, it follows by homogeneity of $V^{\prime}$ that

$$
w_{m}^{\prime \prime}(t)+\varepsilon w_{m}^{\prime}(t)+\left(1-\lambda_{m}\right) w_{m}(t)+\lambda_{m} V^{\prime}\left(w_{m}(t)\right)=\lambda_{m} p(t) /\left|u_{m}\right|_{1}
$$

for $m=1,2, \ldots$. Since $\left|w_{m}\right|_{1}=1$ for $m \geq 1$, it follows from (1.10) that the sequence $\left\{\left|w_{m}^{\prime \prime}\right|_{\infty}\right\}_{1}^{\infty}$ is bounded. Therefore, both of the sequences $\left\{w_{m}(t)\right\}_{1}^{\infty}$ and $\left\{w_{m}^{\prime}(t)\right\}_{1}^{\infty}$ are equicontinuous and uniformly bounded on $(-\infty, \infty)$ so, by Ascoli's lemma, there exists a subsequence $\left\{w_{m_{k}}(t)\right\}_{k=1}^{\infty}$ and a $w \in C_{T}^{1}$ with $|w|_{1}=1$ such that $w_{m_{k}}(t) \rightarrow w(t), w_{m_{k}}^{\prime}(t) \rightarrow w^{\prime}(t)$ as $m \rightarrow \infty$, uniformly on $(-\infty, \infty)$. Since $0 \leq \lambda_{m_{k}} \leq 1$ for all $k \geq 1$, we may assume without loss of generality that $\lambda_{m_{k}} \rightarrow \lambda^{*_{k}} \in[0,1]$ as $k \rightarrow \infty$. Therefore, from (1.10), it follows that the sequence $\left\{w_{m_{k}}^{\prime \prime}(t)\right\}_{1}^{\infty}$ converges uniformly on $(-\infty, \infty)$ so $w$ is of class $C^{2}$ and

$$
w^{\prime \prime}(t)+\varepsilon w^{\prime}(t)+\left(1-\lambda^{*}\right) w(t)+\lambda^{*} V^{\prime}(w(t))=0
$$

Taking the inner product of (1.11) with $w^{\prime}(t)$ and observing that

$$
\int_{0}^{T}\left(w^{\prime}(t), w^{\prime \prime}(t)\right) d t=\frac{1}{2} \int_{0}^{T} \frac{d}{d t}\left|w^{\prime}(t)\right|^{2} d t=0
$$

and

$$
\begin{aligned}
\int_{0}^{T}\left(w^{\prime}(t),\left(1-\lambda^{*}\right) w(t)+\lambda^{*} V^{\prime}(w(t))\right) d t \\
\quad=\int_{0}^{T} \frac{d}{d t}\left[\left(1-\lambda^{*}\right)|w(t)|^{2} / 2+\lambda^{*} V(w(t))\right] d t=0,
\end{aligned}
$$


we find that

$$
\varepsilon \int_{0}^{T}\left|w^{\prime}(t)\right|^{2} d t=0
$$

Hence, $w(t)=\xi=$ constant and according to (1.11)

$$
\left(1-\lambda^{*}\right) \xi+\lambda^{*} V^{\prime}(\xi)=0 .
$$

Taking the inner product of this last equation with $\xi$ and using (1.2), we have

$$
\left(1-\lambda^{*}\right)|\xi|^{2}+2 \lambda^{*} V(\xi)=0 .
$$

Thus, since $0 \leq \lambda^{*} \leq 1$, it follows from $(1.3)^{*}$ that $\xi=0$. Since, this contradicts the fact that $|w|_{1}=1$, the claim that there exists $R$ independent of $u \in C_{T}$ and $\lambda \in[0,1]$ such that (1.7) implies that $|u|_{\infty}<R$ has been established.

From the Leray-Schauder-Shaefer theorem, it follows that for each $\lambda \in[0,1]$ there exists a $u \in C_{T}$ which satisfies (1.7). (See, for example, $[9,1$, p. 61, or 6, p. 71]). In particular since (1.7) has a solution $u$ when $\lambda=1$, it follows that (1.6) has at least one $T$-periodic solution.

Let $\left\{\varepsilon_{m}\right\}_{1}^{\infty}$ be a sequence of positive numbers such that $\varepsilon_{m} \rightarrow 0$ as $m \rightarrow \infty$. By what has been shown for each $m=1,2, \ldots$, there exists $u_{m} \in C_{T}$ such that $u_{m}$ is a solution of (1.6) when $\varepsilon=\varepsilon_{m}$. We claim that the sequence $\left\{\left|u_{m}\right|_{1}\right\}_{1}^{\infty}$ is bounded. Assuming the contrary, we may suppose without loss of generality that $\left|u_{m}\right|_{1} \rightarrow \infty$ as $m \rightarrow \infty$. Setting $z_{m}(t)=u_{m}(t) /\left|u_{m}\right|$, for $m \geq 1$, we have, by positive homogeneity of $V^{\prime}$,

$$
z_{m}^{\prime \prime}(t)+\varepsilon_{m} z_{m}^{\prime}(t)+V^{\prime}\left(z_{m}(t)\right)=p\left((t) /\left|u_{m}\right|_{1}\right.
$$

for $m=1,2, \ldots$. From this it follows that the sequences $\left\{z_{m}(t)\right\}_{1}^{\infty}$ and $\left\{z_{n}^{\prime}(t)\right\}_{1}^{\infty}$ are equicontinuous and uniformly bounded on $(-\infty, \infty)$ so there exists $z \in C_{T}$ with $|z|_{1}=1$ and a subsequence $\left\{z_{m_{k}}(t)\right\}_{1}^{\infty}$ of $\left\{z_{m}\right\}_{1}^{\infty}$ such that $z_{m_{k}}(t) \rightarrow z(t)$ and $z_{m_{k}}^{\prime}(t) \rightarrow z^{\prime}(t)$ as $k \rightarrow \infty$ uniformly with respect to $t \in(-\infty, \infty)$. From (1.12), we infer that the sequence $\left\{z_{m_{k}}^{\prime \prime}(t)\right\}_{1}^{\infty}$ converges uniformly on $(-\infty, \infty)$. Hence $z$ is of class $C^{2}$ and

$$
z^{\prime \prime}(t)+V^{\prime}(z(t))=0 \text {. }
$$

Since $|z|_{1}=1$, this contradicts the assumption that (1.4) has no nontrivial $T$-periodic solution, our claim that the sequence $\left\{\left|u_{m}\right|_{1}\right\}_{1}^{\infty}$ is bounded has been established.

From the differential equation

$$
u_{m}^{\prime \prime}(t)+\varepsilon_{m} u_{m}^{\prime}(t)+V^{\prime}\left(u_{m}(t)\right)=p(t),
$$

it follows that the sequence $\left\{u_{m}^{\prime \prime}(t)\right\}_{1}^{\infty}$ is also uniformly bounded on $(-\infty, \infty)$. Therefore, using the same type of argument used above, we infer the existence of a subsequence of $\left\{u_{m}(t)\right\}_{1}^{\infty}$ such that this subsequence, as well as the corresponding sequences of first and second derivatives converges uniformly on 
$(-\infty, \infty)$. Since the limit of this subsequence is a $T$-periodic solution of (1.5), the proof of the theorem under assumption $(1.3)^{*}$ is complete.

To prove the theorem with $(1.3)^{*}$ replaced by $(1.3)$, we observe that for all sufficiently small $\delta>0$, the system

$$
u^{\prime \prime}(t)+\delta u(t)+V^{\prime}(u(t))=0
$$

has no nontrivial $T$-periodic solution. Indeed, in the contrary case, for $\delta>0$ arbitrarily small, we could find a solution with $C_{T}^{1}$-norm equal to 1 . A compactness argument, similar to those used above, would give a nontrivial solution of (1.4), contradicting one of our hypotheses.

Therefore, since $\delta|x|^{2} / 2+V(x)>0$ for $x \neq 0$, by what we have shown, for small $\delta>0$ there exists a $T$-periodic solution of

$$
u^{\prime \prime}+\delta u+V^{\prime}(u)=p(t) .
$$

The $C_{T}^{1}$-norms of these solutions are bounded as $\delta \rightarrow 0$ since (1.4) has no nontrivial $T$-periodic solution. Therefore, by the same type of compactness argument as used above, we obtain a $T$-periodic solution of (1.5).

Remark. It does not seem possible to prove the theorem more directly by connecting (1.5) rather than (1.6) to a linear equation by a homotopy.

Remark. The theorem remains true if the condition (1.3) is replaced by

$$
x \in \mathbf{R}^{N}, \quad x \neq 0 \Rightarrow V(x)<0 .
$$

In the proof one would consider the parameter-dependent differential equation

$$
u^{\prime \prime}(t)+\varepsilon u^{\prime}(t)-(1-\lambda) u(t)+\lambda V^{\prime}(u(t))=\lambda p(t)
$$

instead of (1.8) and use the fact that the linear differential equation

$$
u^{\prime \prime}(t)+\varepsilon u^{\prime}(t)-u(t)=f(t),
$$

where $\varepsilon>0$, has a unique $T$-periodic solution for any $f \in C_{T}$.

Examples 1. The homogeneous nonlinearity in the differential equation

$$
u^{\prime \prime}(t)+|u(t)|=p(t) \equiv p(t+T),
$$

where $n=1$, does not satisfy the condition (1.3), since in this case $V(x)=$ $\frac{1}{2}(\operatorname{sgn} x) x^{2}$. If $p(t) \neq 0$ and $u(t)$ is a $T$-periodic solution of (1.13), then

$$
\int_{0}^{T}|u(t)| d t=\int_{0}^{T} p(t) d t,
$$

so, in order that (1.13) have a $T$-periodic solution, it is necessary that the mean value of $p$ be positive. If $q(t)$ is a continuous $T$-periodic function with mean value zero, and $p(t)=c+q(t)$ where $c$ is a constant, then by using the wellknown upper and lower solution method for periodic solutions of second-order differential equations (see, for example, [10]) one can easily adapt the methods of [2] (which concerns a boundary value problem for a P.D.E.) to prove the 
existence of $\bar{c}=\bar{c}(q)$ such that (1.13) has a $T$-periodic solution if and only if $c \geq \bar{c}$.

2. Consider the differential equation

$$
u^{\prime \prime}+b u^{+}-a u^{-}=p(t) \equiv p(t+2 \pi),
$$

where $n=1, a>0$ and $b>0$. If $u$ is a nontrivial solution of

$$
u^{\prime \prime}+b u^{+}-a u^{-}=0 \text {, }
$$

then the distance between two consecutive zeros of $u$ which border an interval on which $u$ is positive is $\pi / \sqrt{b}$, since $u^{\prime \prime}+b u=0$ on such an interval. Similarly the distance between two consecutive zeros of $u$ which border an interval on which $u$ is negative is $\pi / \sqrt{a}$. It follows that every nontrivial solution of (1.15) is periodic with least period $\pi / \sqrt{a}+\pi / \sqrt{b}$. Therefore, since the potential for the nonlinearity in (1.15), $V(x)=\left[b\left(x^{+}\right)^{2}+a\left(x^{-}\right)^{2}\right] / 2$, satisfies (1.3), it follows that if

$$
\pi / \sqrt{b}+\pi / \sqrt{a} \neq 2 \pi / m
$$

for $m=1,2, \ldots$, then, for any continuous $2 \pi$-periodic function $p(t),(1.14)$ has a $2 \pi$-periodic solution.

3. Suppose that for some integer $m \geq 1$ we have

$$
\pi / \sqrt{b}+\pi / \sqrt{a}=2 \pi / m
$$

and in addition that

$$
(m-1)^{2}<a<b<(m+1)^{2} .
$$

Let $H$ be the Hilbert space consisting of $2 \pi$-periodic functions defined on $(-\infty, \infty)$ whose restrictions to $[-\pi, \pi]$ belong to $L^{2}[-\pi, \pi]$ with the $L^{2}[-\pi, \pi]$ inner product. Let $W$ be the two-dimensional subspace of $H$ spanned by $\cos m t$ and $\sin m t$ and let $P: H \rightarrow W$ denote orthogonal projection. Since the spectrum of the linear operator $A: D(A) \subset H \rightarrow H$ defined by $A u=-u^{\prime \prime}$ is $\left\{k^{2} \mid k=0,1, \ldots\right\}$ it follows from the Liapunov-Schmidt technique and (1.16) that for any $w_{1} \in W$ there exists a unique $w_{2} \in(I-P) W$ such that

$$
w_{2}^{\prime \prime}+(I-P)\left[b\left(w_{1}+w_{2}\right)^{+}-a\left(w_{1}+w_{2}\right)^{-}\right]=0
$$

and $w_{2}(t+2 \pi) \equiv w_{2}(t)$. (See [5], or the proof of Proposition 2.1 of [3] for more details.) If $u_{0}$ is a nonzero solution of $(1.15)$, then $u_{0}$ is $(2 \pi / m)$-periodic and $P u_{0} \not \equiv 0$, for otherwise, since $w_{2} \equiv 0$ solves $(1.17)$ when $w_{1} \equiv 0$, we would have $(I-P) u_{0}$, and hence $u_{0}$, identically zero.

We claim that if $c^{2}+d^{2} \neq 0$ then there exists no $2 \pi$-periodic solution of

$$
u^{\prime \prime}+b u^{+}-a u^{-}=c \cos m t+d \sin m t \text {. }
$$

Assuming, on the contrary, that there exists a $2 \pi$-periodic solution $u^{*}$ of (1.18), the reasoning used above shows that $P u^{*} \not \equiv 0$. Therefore, since both $P u_{0}$ and $P u^{*}$ are linear combinations of $\cos m t$ and $\sin m t$, both have the form 
$r \sin (m t+\delta)$ for some $r>0$. Therefore there exist numbers $\alpha>0$ and $\gamma$ such that $\hat{u}(t)=\alpha u_{0}(t+\gamma)$, then $P \hat{u}=P u^{*}$. Therefore, since by homogeneity $\hat{u}$ is also a solution of (1.15), it follows that if $w_{1}=P \hat{u}$, then both $w_{2}=(I-P) \hat{u}$ and $w_{2}=(I-P) u^{*}$ solve $(1.17)$, so by uniqueness $(I-P) \hat{u}=(I-P) u^{*}$. Therefore we have the absurdity $\hat{u}=u^{*}$. This contradiction proves the claim.

This phenomenon is, of course, well known as resonance in the linear case $a=b=m^{2}$.

The work of Podolak [8] shows that if $n=1$ and periodic boundary conditions are replaced by Dirichlet boundary conditions, then the statement of the theorem does not remain true.

\section{REFERENCES}

1. K. Deimling, Nonlinear functional analysis, Springer-Verlag, Berlin, Heidelberg and New York, 1985.

2. J. L. Kazdan and F. W. Warner, Remarks on some quasilinear elliptic equations, Comm. Pure Appl. Math. 28 (1975), 567-597.

3. A. C. Lazer and P. J. McKenna, Critical point theory and boundary value problems with nonlinearities crossing multiple eigenvalues. II, Comm. in Partial Differential Equations 11 (1986), 1653-1676.

4. __ Large scale oscillatory behaviour in loaded asymmetric systems, Ann. Inst. Henri Poincaré 4 (1987), 243-276.

5. __ A symmetry theorem and applications to nonlinear partial differential equations, J. Differential Equations 72 (1988), 95-106.

6. N. G. Lloyd, Degree theory, Cambridge Univ. Press, London and New York, 1978.

7. P. J. McKenna and W. Walter, Nonlinear oscillations in a suspension bridge, Arch. Rat. Mech. Anal. 98 (1987), 168-177.

8. E. Podolak, On the range of operator equations with an asymptotically nonlinear term, Indiana Univ. Math. J. 25 (1976), 1127-1137.

9. H. Schaefer, Uber die Methode der a priori Schranken, Math. Ann. 29 (1955), 415-416.

10. K. Schmitt, Periodic solutions of nonlinear second order ordinary differential equations, Math. Z. 98 (1967), 200-207.

Added in Proof. The result in Example 2 is also in:

11. E. N. Dancer, On the Dirichlet problem for weakly nonlinear elliptic partial differential equations, Proc. Roy. Soc. Edinburgh, Sec. A 76 (1977), 283-300.

Department of Mathematics and Computer Science, University of Miami, Coral GABLES, Florida 33124

Department of Mathematics, University of Connecticut, Storrs, Connecticut 06268 